\title{
On the derivation of some fundamental expressions for the average stress tensor in systems of interacting particles
}

\author{
R. J. J. Jongschaap \\ Rheology Group, Department of Applied Physics, Twente University of Technology, Enschede (The Netherlands)
}

\begin{abstract}
The so-called generalized Kramers-Kirkwood expression for the average stress tensor of a system of interacting point particles, derived by Bird and Curtiss on using a phase-space-kinetic formalism has been reconsidered from different points of view. First a derivation based upon volume averaging is discussed, and after that a derivation based upon a virtual work principle. The latter approach offers the possibility of distinguishing reversible (including thermodynamic and Brownian) and dissipative forces and stresses by using a projection operator, associated with the constraints of the system.
\end{abstract}

Key words: Average stress tensor, interacting particles, volume averaging, virtual work principle, constraint

\section{Introduction}

In a previous paper [1] the derivation of some expressions for the average stress tensor in concentrated systems of interacting particles of an arbitrary shape was discussed from various points of view. In the present paper we will focus our attention upon systems consisting of interacting point particles. The general expressions for the average stress tensor for such systems have been reviewed recently by Bird and Curtiss [2]. Their most fundamental expression is the so-called generalized Kramers-Kirkwood expression, which is valid for arbitrary mixtures of species $\alpha=1,2,3, \ldots$, modelled by bead-rod-spring systems, and reads (in our notation):

$$
\tilde{\boldsymbol{T}}=n \sum_{i \alpha \beta} x_{\beta} N_{\beta}\left\langle\overline{\boldsymbol{f}}_{i \alpha \beta}^{I}\left(\boldsymbol{r}_{i \alpha}-\boldsymbol{r}_{\alpha}\right)\right\rangle .
$$

Here $n$ is the total density of molecules of all species, $x_{\beta}$ is the mole fraction of species $\beta, N_{\beta}$ is the number of beads in the mechanical model representing a molecule of species $\beta, \bar{f}_{i \alpha \beta}^{I}$ is the force on bead $i$ of a molecule of species $\alpha$ due to the molecules of species $\beta$, including $\beta=\alpha$, averaged with respect to all configurations of these molecules, $\boldsymbol{r}_{i \alpha}$ is the position of bead $i$ of species $\alpha, r_{\alpha}$ the center of a molecule of species $\alpha$ and the brackets denote an average with respect to the internal configurations of the molecules of species $\alpha$. On per208 forming the summation with respect to $\beta$ eq. (1) may also be written as:

$$
\tilde{\boldsymbol{T}}=\sum_{i \alpha} n_{\alpha}\left\langle\overline{\boldsymbol{f}}_{i \alpha}^{l^{\prime}}\left(\boldsymbol{r}_{i \alpha}-\boldsymbol{r}_{\alpha}\right)\right\rangle
$$

in which $\overline{\boldsymbol{f}}_{i \alpha}^{I^{\prime}}$ is the total external force on bead $i$ of a molecule of species $\alpha$ due to the interactions with all other molecules, i.e.

$$
\overline{\boldsymbol{f}}_{i \alpha}^{I^{\prime}}=\sum_{\beta} x_{\beta} N_{\beta} \overline{\boldsymbol{f}}_{i \alpha \beta}^{I} \text {, and } n_{\alpha} \text { is number density of }
$$

molecules of species $\alpha$. So the macroscopical stress tensor $\tilde{\boldsymbol{T}}$ may be considered as a weighted sum $\tilde{\boldsymbol{T}}=\sum_{\alpha} n_{\alpha} \tilde{\boldsymbol{T}}_{\alpha}$ of partial stresses

$$
\tilde{\boldsymbol{T}}_{\alpha}=\frac{1}{V} \sum_{i}\left\langle\overline{\boldsymbol{f}}_{i \alpha}^{I^{\prime}}\left(\boldsymbol{r}_{i \alpha}-\boldsymbol{r}_{\alpha}\right)\right\rangle
$$

representing the contributions of the individual molecules of species $\alpha$.

In [2] it has been shown that eq. (1) is sufficiently general that all the well-known dilute solution expressions, including the so-called Kramers and Giesekus forms for flexible and rigid macromolecules, and also the formula used in the Curtiss-Bird theory for polymer melts [3], can be derived from it. Despite its simple appearance, the derivation of this expressions is rather complicated. This derivation is given in [4] and 
rests entirely on the phase-space-kinetic theory of polymeric fluids, developed by Curtiss, Bird and Hassager [5]. In this derivation the macroscopic equations of change of the continuum are derived by averaging the microscopic equations of motion over an ensemble of macroscopically identical systems. From the macroscopic balance equation of momentum the expression (1) is obtained.

Although this procedure offers a firm basis for this result, and the approximations needed to derive it are made explicit, it is still unsatisfactory as a derivation of eq. (1) since the final result is obtained after very lengthy and tedious algebraic manipulations with generalized coordinates in the statistic mechanical formalism. Noting that the final result (1) or equivalently (3) is of a very simple shape with no generalized coordinates and only an average in configuration space, we surmise that in addition to the original derivation just mentioned other, more direct, derivations should be possible. In the present paper it will be shown that this is the case indeed. In the next section a derivation based upon the kind of volume averaging, discussed in [1], will be given and in section 3 a derivation based upon a principle of virtual work is presented. The latter derivation offers'some interesting features in the discussion of systems with constraints, especially in the distinction between thermodynamic stresses and dissipative stresses in such type of systems. These items will be discussed further in section 4 , in connection with a specific example.

\section{Volume averaging}

A simple and direct way to relate the macroscopical stress tensor to microscopical stresses and forces is the method of volume averaging. In this method which is commonly used in dispersion rheology $[6,7]$ the contribution $\tilde{T}_{i}$ to the macroscopical stress of a particle $i$ is identified with the volume average

$$
\tilde{T}_{i}=\frac{1}{V} \int_{V_{i}} \boldsymbol{T} d V
$$

in which $T$ is the microscopic stress field inside the particle and $V$ a macroscopically small averaging volume. On using the balance equation $\operatorname{div} \boldsymbol{T}=\mathbf{0}$ the volume integral can be transformed into a surface integral and the following expression is obtained:

$$
\tilde{\boldsymbol{T}}_{i}=\frac{1}{V} \int_{\partial V_{i}} \boldsymbol{T} \cdot \boldsymbol{n}\left(\boldsymbol{r}-\boldsymbol{r}_{i}\right) d S .
$$

This expression in which $\boldsymbol{r}_{i}$ is an arbitrary point, is valid for non-interacting particles of any shape. Usually the stresses $\boldsymbol{T} \cdot \boldsymbol{n}$ on $\partial V_{i}$ are due to the hydrodynamic forces acting upon the particle.

In systems of interacting particles the method of volume averaging can still be used if instead of the microscopical stress field $\boldsymbol{T}$ the field $\boldsymbol{T}+\boldsymbol{T}^{\boldsymbol{I}}$ is used, in which $\boldsymbol{T}^{I}$ is a fictitious stress field describing the interactions between the particles. This method, which was discussed extensively in [1] yields for the contribution to the macroscopical stress tensor of a set of particles in a volume $V$ :

$$
\tilde{\boldsymbol{T}}=\frac{1}{V} \int_{i \in V \partial V_{i}} T \cdot \boldsymbol{n}\left(\boldsymbol{r}-\boldsymbol{r}_{i}\right) d S+\frac{1}{2 V_{i, j \in V}} \sum_{j} \boldsymbol{f}_{i j}^{I} \boldsymbol{r}_{i j} .
$$

In this expression $\boldsymbol{r}_{i}$ is an arbitrary point inside particle $i, f_{i j}^{I}$ is the interaction force on particle $i$ due to particle $j$ and $\boldsymbol{r}_{i j}=\boldsymbol{r}_{j}-\boldsymbol{r}_{i}$. In the derivation of (7) the interactions were assumed to be short-range, in comparison with the dimensions of the volume $V$. For point particles (7) reduces to:

$$
\tilde{\boldsymbol{T}}^{I}=\frac{1}{2 V} \sum_{i, j \in V} \boldsymbol{f}_{i j}^{I} \boldsymbol{r}_{i j}
$$

If one wishes to avoid the use of the fictitious stress field $\boldsymbol{T}^{I}$, the interactions between the particles can also be represented by connecting rods (connectors) with elastic properties determined by the interaction potentials. In that case eq. (5) can be applied to the whole networkstructure. To this end the volume $V$ is devided into cells $i$ such that each cell contains yust one particle and each connector crosses the common boundary of the cells around the particles at its terminal points. The total stress $\tilde{T}^{I}$ due to all cells in $V$ can be written then as $\tilde{\boldsymbol{T}}^{I}=\sum_{i} \tilde{\boldsymbol{T}}_{i}^{I}$ in which $\tilde{\boldsymbol{T}}_{i}^{I}$ is the contribution of cell $i$. This contribution, in turn, can be written as $\tilde{\boldsymbol{T}}_{i}^{I}=\sum_{j} \tilde{\boldsymbol{T}}_{i j}^{I}$ in which $\tilde{\boldsymbol{T}}_{i j}^{I}$ is the contribution of the surface $S_{i j}$ connecting the cells $i$ and $j$ to the surface integral in (5), so:

$$
\begin{aligned}
& \tilde{\boldsymbol{T}}_{i j}^{I}=\frac{1}{V} \int_{S_{i j}} \boldsymbol{T} \cdot \boldsymbol{n}\left(\boldsymbol{r}-\boldsymbol{r}_{i}\right) d S, \\
& \tilde{\boldsymbol{T}}_{j i}^{I}=\frac{1}{V} \int_{S_{i j}} \boldsymbol{T} \cdot(-\boldsymbol{n})\left(\boldsymbol{r}-\boldsymbol{r}_{j}\right) d S .
\end{aligned}
$$

In these expressions $\boldsymbol{T}$ is the stress in the connector between the particles $i$ and $j$ and the unit normal $\boldsymbol{n}$ is external with respect to the cell around the particle $i$.

Since

$$
\int_{S_{i j}} \boldsymbol{T} \cdot \boldsymbol{n} d S=\boldsymbol{f}_{i j}^{I}
$$


it follows from (9) that

$$
\tilde{\boldsymbol{T}}_{i j}^{I}+\tilde{\boldsymbol{T}}_{j i}^{I}=\frac{1}{V} \boldsymbol{f}_{i j}^{I} \boldsymbol{r}_{i j}
$$

So the total stress $\tilde{\boldsymbol{T}}^{I}$ becomes

$$
\tilde{\boldsymbol{T}}^{I}=\sum_{i} \tilde{\boldsymbol{T}}_{i}^{I}=\sum_{i j} \tilde{\boldsymbol{T}}_{i j}^{I}=\frac{1}{2} \sum_{i j}\left(\tilde{\boldsymbol{T}}_{i j}^{I}+\tilde{\boldsymbol{T}}_{j i}^{I}\right)=\frac{1}{2 V} \sum_{i j} \boldsymbol{f}_{i j}^{I} \boldsymbol{r}_{i j}
$$

in accordance with (8). Note that in this derivation no distinction was made between particles in and outside $V$. In fact the terms $\boldsymbol{T}_{i j}^{I}$ with $i \in V$ and $j \notin V$ should be treated separately, since in that case, as $j$ lies outside the averaging volume $V, \tilde{\boldsymbol{T}}_{j i}^{I}$ does not contribute to $\tilde{\boldsymbol{T}}^{I}$. This effect however can be neglected since, because of the assumption of short range forces, the contribution of such terms in (11) to the total stress tensor $\tilde{\boldsymbol{T}}$ will be relatively small. A similar approximation was made [1] in the derivation of eq. (7).

A third way of obtaining eq. (8) is based upon the expression

$$
\tilde{\boldsymbol{T}}^{I}(\boldsymbol{r})=\frac{1}{2} \sum_{i j}^{1} \int_{0}^{1}\left\langle\boldsymbol{f}_{i j}^{I} \boldsymbol{r}_{i j} \delta\left(\boldsymbol{r}_{i}-\boldsymbol{r}+\xi \boldsymbol{r}_{i j}\right)\right\rangle d \xi
$$

which can be derived [8] by identifying the average of the forces $f_{i j}$ between two particles $i$ and $j$ for all configurations in which a straight line through the particles crosses a surface element $d S$, with the average traction $\tilde{\boldsymbol{t}}^{I}=\tilde{\boldsymbol{T}}^{I} \cdot \boldsymbol{n}$ transmitted through $d S$. On using again the assumption of short range forces we obtain from (12)

$$
\tilde{\boldsymbol{T}}^{I}(\boldsymbol{r})=\frac{1}{2} \sum_{i j}\left\langle\boldsymbol{f}_{i j}^{I} \boldsymbol{r}_{i j} \delta\left(\boldsymbol{r}_{i}-\boldsymbol{r}\right)\right\rangle .
$$

We now take the volume average of this expression. If the averaging volume is large enough the ensemble averaging is automatically performed on taking the volume average and may be omitted, so

$$
\tilde{\boldsymbol{T}}^{I}=\frac{1}{2 V} \sum_{\substack{i, j \\ i \in \boldsymbol{V}}} f_{i j}^{I} \boldsymbol{r}_{i j}
$$

In this expression the particle $j$ may lie outside $V$ but, as before, we only consider the terms for which $i, j \in V$, and again the result (8) is obtained.

We now return to the derivation of the generalized Kramers-Kirkwood expression (3). To this end we rewrite (8) as follows:

$$
\begin{aligned}
\tilde{\boldsymbol{T}}^{I} & =\frac{1}{2 V} \sum_{i, j \in V} f_{i j}^{I} \boldsymbol{r}_{i j}=\frac{1}{2 V} \sum_{i, j \in V} f_{i j}^{I}\left(\boldsymbol{r}_{j}-\boldsymbol{r}_{i}\right) \\
& =\frac{-1}{2 V} \sum_{i, j \in V}\left(f_{j i}^{I} \boldsymbol{r}_{j}+\boldsymbol{f}_{i j}^{I} \boldsymbol{r}_{i}\right)=\frac{-1}{V_{i, j}} \sum_{i V} f_{i j}^{I} \boldsymbol{r}_{i}=\frac{-1}{V} \sum_{i} f_{i}^{I V} \boldsymbol{r}_{i}
\end{aligned}
$$

in which

$$
f_{i}^{I V}=\sum_{j \in V} f_{i j}^{I}
$$

equals the force on particle $i \in V$ due to the interactions with all other particle $j \in V$. (Note the similarity of the expressions (14) and (15) to (4) and (5) respectively).

So far we only took into account forces due to the interactions between the particles. Since we are dealing however with a description in the configuration space, in which rapidly fluctuating microscopical velocities of the particles have been averaged out, additional forces due to the momentum flux associated with these microscopial velocities have to be introduced. These forces, usually called Brownian forces are governed by the configurational distribution function $\psi\left(\boldsymbol{r}_{i} \ldots \boldsymbol{r}_{N}, t\right)$ of the particles and tend to change a nonhomogeneous distribution in configuration space into a homogeneous one. The actual equilibrium distribution of the system is determined by the combined action of interaction forces, external forces and Brownian forces. The wellknown expression for the Brownian forces in a system of point particles reads:

$$
f_{i}^{B}=-k T \frac{\partial}{\partial r_{i}} \ln \psi .
$$

In the case of constraints on the motion of the particles such as a fixation of the distances between some of the particles, usually represented by rigid rods in breadrod-spring models, eq. (17) has to be modified. This can be done [9] by a transformation to generalized coordinates, or [10] by regarding the $\boldsymbol{r}_{i}$ in (17) as independent coordinates and taking into account the constraints by adding an appropriate constraining force in the right-hand side of (17), or equivalently by taking the orthogonal projection of (17) into the subspace of the configuration space to which the motion is constrained. In the latter approach in general we have

$$
\boldsymbol{f}_{i}^{B}=-k T \sum_{j} \boldsymbol{P}_{i j} \cdot \frac{\partial}{\partial \boldsymbol{r}_{j}} \ln \psi
$$

The tensors $\boldsymbol{P}_{i j}$ determine an orthogonal projection operator given by

$$
\boldsymbol{P}_{i j}=\delta_{i j} \mathbf{1}-\sum_{\alpha} \frac{\partial C_{\alpha}}{\partial \boldsymbol{r}_{i}} \frac{\partial C_{\alpha}}{\partial \boldsymbol{r}_{j}} /\left|\frac{\partial C_{\alpha}}{\partial \boldsymbol{r}_{i}}\right|\left|\frac{\partial C_{\alpha}}{\partial \boldsymbol{r}_{j}}\right|
$$

in which the $C_{\alpha}$ are functions, determining the constraints by the equations

$$
C_{\alpha}\left(r_{1}, r_{2} \ldots r_{N}\right)=0 \text {. }
$$

If the distribution function $\psi$ is defined only in the constrained space any arbitrary extension of $\psi$ to the 
whole coordinate space may be used in (18), since all of these functions will give the same results for $f_{i}^{B}$.

In section 4 an example will be discussed in which the formalism outlined above will be applied. For our purpose however we only need to know that for a given configurational distribution function of the system that means for any macroscopically defined (nonequilibrium-)state of the system - with any configuration of the particles a well defined set of Brownian forces, acting upon the particles is associated. These forces could in principle be taken into account by a procedure of volume averaging by the introduction of a fictitious stress field $\boldsymbol{T}^{B}$ analogous to the field $\boldsymbol{T}^{I}$ discussed in [1]. This however leads to conceptual difficulties because of the assignment of appropriate boundary conditions for $\boldsymbol{T}^{B}$. Instead, in our present investigation the effect of Brownian forces will be treated by noting that since these forces are determined by the configuration of the particles in $V$ their effect on the stress $\tilde{\boldsymbol{T}}$ due to these particles will be similar to the effect of the forces $f_{i}^{I V}$ in eq. (15), so the inclusion of the Brownian contribution is achieved by the addition of an extra-term in (15):

$$
\tilde{\boldsymbol{T}}=-\frac{1}{V} \sum_{i}\left(\boldsymbol{f}_{i}^{I V}+\boldsymbol{f}_{i}^{B}\right) \boldsymbol{r}_{i}
$$

On using the equilibrium of forces on the particles

$$
f_{i}^{I V}+f_{i}^{I V^{\prime}}+f_{i}^{B}=\mathbf{0}
$$

in which $f_{i}^{I V^{\prime}}$ is the total force on particle $i$ due to influences from outside $V$, we also have

$$
\tilde{\boldsymbol{T}}=\frac{1}{V} \sum_{i} \boldsymbol{f}_{i}^{I V^{\prime \prime}} \boldsymbol{r}_{i}
$$

Eqs. (21) and (23) form the basis for our derivation of the Kramers-Kirkwood expression (3). To this end the particles in $V$ will be subdivided into different species $\alpha, \beta, \ldots$ consisting of clusters $a, b \ldots$ (molecules) of particles, $i, j, \ldots$ The position vector of, and the force on a particle $i$ of cluster $a$, of the species $\alpha$ will be denoted by $\boldsymbol{r}_{i a \alpha}$ and $\boldsymbol{f}_{i a \alpha}$ respectively. The force equilibrium (22) now becomes

$$
\boldsymbol{f}_{i a \alpha}^{I V}+\boldsymbol{f}_{i a \alpha}^{I V^{\prime}}+\boldsymbol{f}_{i a \alpha}^{B}=\mathbf{0}
$$

from which we obtain

$$
\sum_{i a \alpha}\left(f_{i a \alpha}^{I V} \boldsymbol{r}_{i a \alpha}+\boldsymbol{f}_{i a \alpha}^{I V^{\prime}} \boldsymbol{r}_{i a \alpha}+\boldsymbol{f}_{i a \alpha}^{B} \boldsymbol{r}_{i a \alpha}\right)=\mathbf{0}
$$

and also

$$
\sum_{i a \alpha}\left(f_{i a \alpha}^{I V} \boldsymbol{r}_{a \alpha}+f_{i a \alpha}^{I V^{\prime}} \boldsymbol{r}_{a \alpha}+\boldsymbol{f}_{i a \alpha}^{B} \boldsymbol{r}_{a \alpha}\right)=\mathbf{0} .
$$

Here

$$
\boldsymbol{r}_{a \alpha}=\frac{1}{N} \sum_{i} \boldsymbol{r}_{i a \alpha} \quad(i \in V)
$$

designates the centre of molecule $a$ of species $\alpha$.

From (23) we have

$$
\tilde{T}=\frac{1}{V} \sum_{i a \alpha} f_{i a \alpha}^{I V^{\prime \prime}} \boldsymbol{r}_{i a \alpha}
$$

which, on using (27) may also be written as

$$
\tilde{\boldsymbol{T}}=\frac{1}{V} \sum_{i a \alpha} \boldsymbol{f}_{i a \alpha}^{I V^{\prime \prime}}\left(\boldsymbol{r}_{i a \alpha}-\boldsymbol{r}_{a \alpha}\right)-\frac{1}{V} \sum_{a \alpha}\left(\boldsymbol{f}_{a \alpha}^{I V} \boldsymbol{r}_{a \alpha}+\boldsymbol{f}_{a \alpha}^{B} \boldsymbol{r}_{a \alpha}\right)
$$

in which

$$
f_{a \alpha}^{I V}=\sum_{i} f_{i a \alpha}^{I V}
$$

and

$$
f_{a \alpha}^{B}=\sum_{i} f_{i a \alpha}^{B} .
$$

The Brownian term in (30) may also be written as

$$
\begin{aligned}
\frac{1}{V} \sum_{a \alpha} \boldsymbol{f}_{a \alpha}^{B} \boldsymbol{r}_{a \alpha}= & \frac{1}{V} \sum_{i a \alpha} \boldsymbol{f}_{i a \alpha}^{B} \boldsymbol{r}_{i a \alpha} \\
& -\frac{1}{V} \sum_{i a \alpha} \boldsymbol{f}_{i a \alpha}^{B}\left(\boldsymbol{r}_{i \alpha \alpha}-\boldsymbol{r}_{a \alpha}\right)
\end{aligned}
$$

i. e. the total contribution of all particles minus an internal contribution due to the motion of the particles $i a \alpha$ with respect to the centre of the molecule $a \alpha$ to which they belong.

The term in (30) containing $\boldsymbol{f}_{a \alpha}^{I V} \boldsymbol{r}_{a \alpha}$ can - in analogy with (15) - be written as:

$$
\frac{1}{V} \sum_{\alpha} \boldsymbol{f}_{a \alpha}^{I V} \boldsymbol{r}_{a \alpha}=-\frac{1}{2 V} \sum_{a \alpha b \beta} \boldsymbol{f}_{a \alpha b \beta}^{I V} \boldsymbol{r}_{a \alpha b \beta}
$$

in which

$$
\boldsymbol{r}_{a \alpha, b \beta}=\boldsymbol{r}_{b \beta}-\boldsymbol{r}_{a \alpha}
$$

and

$$
f_{a \alpha b \beta}^{I V}=\sum_{\substack{i j \in V \\ a \neq b}} f_{i a \alpha j b \beta}^{I V}
$$

where $f_{i a \alpha j b \beta}^{I V}$ is the force on particle $i a \alpha$ due to the particles in $V$ of the molecules $b \neq a$ of species $\beta$ (including $\beta=\alpha$ ). In the case of short range interactions the terms in the summation (34) will only differ from zero if

$$
\boldsymbol{r}_{a \alpha, b \beta} \approx\left(\boldsymbol{r}_{i a \alpha}-\boldsymbol{r}_{a \alpha}\right)-\left(\boldsymbol{r}_{j b \beta}-\boldsymbol{r}_{b \beta}\right) .
$$


In that case on substituting (35) and (36) in (34) and using the fact that $f_{a \alpha b \beta}^{I V}=-f_{b \beta a \alpha}^{I V}$ we obtain

$$
\frac{1}{V} \sum_{a \alpha} \boldsymbol{f}_{a \alpha}^{I V} \boldsymbol{r}_{a \alpha}=\sum_{i a \alpha} \boldsymbol{f}_{i a \alpha}^{I V a^{\prime}}\left(\boldsymbol{r}_{i a \alpha}-\boldsymbol{f}_{a \alpha}\right)
$$

in which

$$
f_{i a \alpha}^{I V a^{\prime}}=\sum_{j \beta} f_{i a \alpha j b \beta}^{I V}
$$

is the force on the particle $i$ of molecule $a$ of the species $\alpha$ due to all particles in $V$ not belonging to molecule $a$. From (30), (33) and (37) we finally obtain

$$
\tilde{\boldsymbol{T}}=\tilde{\boldsymbol{T}}^{B}-\tilde{\boldsymbol{T}}^{B, r}+\frac{1}{V} \sum_{i a \alpha} \boldsymbol{f}_{i a \alpha}^{I a^{\prime}}\left(\boldsymbol{r}_{i a \alpha}-\boldsymbol{r}_{a \alpha}\right)
$$

in which the Brownian part $\tilde{T}^{B}-\tilde{T}^{B, r}$ is given by the negative right hand side of (33) and $f_{i a x}^{I a^{\prime}}$ the total force on the particle $i \alpha$ of molecule $a$ due to interactions with other molecules in and outside $V$. Since $V$ was assumed to be large as compared to the molecular dimensions the summation with respect to $a$ in (39) will consist of a large number of terms and may be changed into an ensemble average.

In this way we obtain

$$
\tilde{\boldsymbol{T}}=\tilde{\boldsymbol{T}}^{B}-\tilde{\boldsymbol{T}}^{B, r}+\sum_{i \alpha} n_{\alpha}\left\langle\overline{\boldsymbol{f}}_{i \alpha}^{I^{\prime}}\left(\boldsymbol{r}_{i \alpha}-\boldsymbol{r}_{\alpha}\right)\right\rangle
$$

where we also have preaveraged the force on the particle $i \alpha$. The result (40) is equivalent to the Kramers-Kirkwood formula (2), in which the Brownian terms were neglected. (The complete expression, including the Brownian terms was given in [4]). So we see that this formula can be considered as a special case of the simple formulae (21) and (23) which in turn can be obtained by methods of volume averaging.

Since eqs. (21) and (23) appear to play a fundamental role in the derivation of expressions of the stress tensor they will be considered from another point of view in the next section.

\section{Thermodynamic considerations}

Let us consider a set of interacting particles in a region $V$ and suppose that this system is in equilibrium with external forces $\boldsymbol{f}_{i}^{I V^{\prime \prime}}$ applied on the particles from outside the region $V$. If there are no constraints upon the motion of the particles we may consider a small homogeneous deformation in which the motion of all particles is determined by the infinitesimal displace- ment gradient tensor

$$
\delta \boldsymbol{H}=\frac{\partial \delta \boldsymbol{r}}{\partial \boldsymbol{r}} .
$$

The work supplied to the system then becomes

$$
\delta W=\sum_{i} \boldsymbol{f}_{i}^{I V^{\prime \prime}} \cdot \delta \boldsymbol{r}_{i}=\sum_{i} \boldsymbol{f}_{i}^{I V^{\prime \prime}} \boldsymbol{r}_{i}: \delta \boldsymbol{H} .
$$

Since in general the work supplied to a volume $V$ of a continuous medium in an infinitesimal deformation is given by

$$
\delta W=V \tilde{\boldsymbol{T}}: \boldsymbol{\delta} \boldsymbol{H}
$$

we immediately obtain in this case the result (23) by equating (42) and (43) for arbitrary values of $\delta W$. If during the supply of work the applied forces are in equilibrium with the internal forces in the system of particles all work will be reversibly stored in the system and the stress tensor $\tilde{\boldsymbol{T}}$ is also given by a derivation of an appropriate thermodynamic potential (under isothermal conditions: the free energy).

In systems with constraints the procedure outlined above cannot be applied in the same way, since in that case, because of the constraints, the particles cannot follow affinely a homogeneous motion determined by a displacement gradient $\boldsymbol{\delta} \boldsymbol{H}$. So a reversible storage of energy in the system by such type of motion is impossible. On the other hand, in the constrained system, in equilibrium with a particular set of external forces $f_{i}^{I V^{\prime}}$, the internal forces between the particles and so also the stress will be the same as in an unconstrained system in equilibrium with the same set of forces. Therefore in the constrained system the stress tensor can be calculated in the same way as before by considering $\delta W$ as the virtual work corresponding to a given set of external forces under the virtual displacements $\delta r_{i}$ which in general will violate the constraints. So also in the case of a constrained system we arrive at the result (23).

In eq. (19) a general projection operator was defined by which the constraints are characterized. This projection operator can be used to split the stress tensor $\tilde{\boldsymbol{T}}$ in a reversible part $\tilde{\boldsymbol{T}}^{R}$ and a dissipative part $\tilde{\boldsymbol{T}}^{D}$. To this end we consider the set vectors $\left\{\delta \boldsymbol{r}_{i}\right\}$ as a vector in configuration space and decompose this vector in a component in, and a component perpendicular to the constraint space:

$$
\delta \boldsymbol{r}_{i}=\sum_{j} \boldsymbol{P}_{i j} \cdot \delta \boldsymbol{r}_{j}+\sum_{j}\left(\delta_{i j} \mathbf{1}-\boldsymbol{P}_{i j}\right) \cdot \delta \boldsymbol{r}_{j}
$$

If this form is substituted in the expression of $\delta W$ we get two terms, the first one gives the work due to a deformation obeying the constraints and the second 
one due to a virtual motion violating the constraints. Since the components of the external forces which lie in the constraint space are in equilibrium with internal forces by which reversible storage of energy is possible the first term in $\delta W$ will be called the reversible part of the virtual work and

$$
\tilde{\boldsymbol{T}}^{R}=\frac{1}{V} \sum_{i j} \boldsymbol{f}_{i}^{I V^{\prime}} \cdot \boldsymbol{P}_{i j} \boldsymbol{r}_{j}=\frac{1}{V} \sum_{i j} \boldsymbol{P}_{i j} \cdot \boldsymbol{f}_{i}^{I V^{\prime}} \boldsymbol{r}_{j}
$$

the reversible stress tensor. (In the last step use has been made of the fact that since the projection is orthogonal, the projection operator is symmetric.) On the other hand since the second term in (44) is perpendicular to the constraint space no storage of work is associated with this part of the virtual motion. Therefore the corresponding term in $\delta W$ will be considered as dissipative and

$$
\tilde{\boldsymbol{T}}^{D}=\frac{1}{V} \sum_{i j}\left(\delta_{i j} \mathbf{1}-\boldsymbol{P}_{i j}\right) \cdot \boldsymbol{f}_{i}^{I V^{\prime}} \boldsymbol{r}_{j}
$$

will be called the dissipative stress tensor. The total stress $\boldsymbol{T}=\boldsymbol{T}^{R}+\boldsymbol{T}^{D}$ is still given by (23).

The reversible stress tensor $\boldsymbol{T}^{R}$ can be derived from an appropriate thermodynamic potential. In isothermal processes this is the free energy $A$, which for a state characterized by a nonequilibrium configurational distribution function $\psi(\{\boldsymbol{r}\}, t)$ is given by the expression [11]:

$$
A=\int(k T \psi \ln \psi+\psi \varphi) d\{\boldsymbol{r}\}
$$

in which $\varphi$ is an interaction potential such that $\sum_{j} \boldsymbol{P}_{i j} \cdot \frac{\partial \varphi}{\partial \boldsymbol{r}_{j}}=\boldsymbol{f}_{i}^{I R V}$, the force on particle $i$ due to interactions (no constraint forces) with all other particles in $V$. Since in equilibrium

$$
\psi_{0}=\left(\exp -\frac{\varphi}{k T}\right) / \int \exp -\frac{\varphi}{k T} d\{\boldsymbol{r}\}
$$

and

$$
A_{0}=-k T \ln \int \exp -\frac{\varphi}{k T} d\{\boldsymbol{r}\}
$$

eq. (47) can also be written as

$$
A=A_{0}+k T \int \psi \ln \left(\psi / \psi_{0}\right) d\{\boldsymbol{r}\} .
$$

This expression is equivalent to the Boltzmann entropy postulate (see for instance [12]) of statistical mechanics for the entropy of a non-equilibrium state characterized by a distribution function $\psi$ :

$$
S=S_{0}-k \int \psi \ln \left(\psi / \psi_{0}\right) d\{\boldsymbol{r}\} .
$$

The equivalence of (48) and (49) follows by considering $s=-k \ln \left(\psi / \psi_{0}\right)$ as a local density of entropy in configuration space and noting that since the specific internal energy $u$ only depends upon $V(\{r\})$ the local change in the density $a=u-T s$ of free energy becomes $a-a_{0}=-T\left(s-s_{0}\right)$.

For the change of free energy corresponding to a change $\delta \psi$ of the distriution function we obtain from (48):

$$
\delta A=k T \int\left[1+\ln \left(\psi / \psi_{0}\right)\right] d\{\boldsymbol{r}\} \delta \psi .
$$

So the quantity

$$
\mu=k T\left[1+\ln \left(\psi / \psi_{0}\right)\right]
$$

can be considered [13] as a local chemical potential in configuration space. From this quantity a thermodynamic (or reversible) force $\left\{f^{R}\right\}$ in configuration space can be derived:

$$
\left\{\boldsymbol{f}^{R}\right\}=-\frac{\partial \mu}{\partial\{\boldsymbol{r}\}}=-k T \frac{\partial}{\partial\{\boldsymbol{r}\}} \ln \left(\frac{\psi}{\psi_{0}}\right)
$$

(for unconstrained systems), by which, due to the tendency of the free energy of the system to seek a minimum value, the system is driven to the equilibrium distribution. The decomposition of $\left\{f^{R}\right\}$ into three-dimensional components gives the thermodynamic forces on the particles, including Brownian forces and interaction forces.

For constrained systems, similar to eq. (18) we have

$$
\begin{aligned}
\boldsymbol{f}_{i}^{R} & =-\sum_{i} \boldsymbol{P}_{i j} \cdot \frac{\partial \mu}{\partial \boldsymbol{r}_{i}} \\
& =-k T \sum_{j} \boldsymbol{P}_{i j} \cdot \frac{\partial}{\partial \boldsymbol{r}_{j}} \ln \psi+k T \sum_{j} \boldsymbol{P}_{i j} \cdot \frac{\partial}{\partial \boldsymbol{r}_{j}} \ln \psi_{0} \\
& =\boldsymbol{f}_{i}^{B}+\boldsymbol{f}_{i}^{I R V},
\end{aligned}
$$

in which $f_{i}^{B}$ is the Brownian force, defined in eq. (18) and $f_{i}^{I R V}$ the interaction force discussed just below eq. (47).

The orthogonal projections of the external forces $f_{i}^{I V^{\prime}}$ into the constraint space are in equilibrium with the reversible force $f_{i}^{R}$ and perpendicular to the constraining forces $f_{i}^{I D V}$ in the system, so we have: $f_{i}^{I V^{\prime \prime}}+f_{i}^{R}+f_{i}^{I D V}=\mathbf{0}$, in which

$$
\boldsymbol{f}_{i}^{R}=-\sum_{j} \boldsymbol{P}_{i j} \cdot \boldsymbol{f}_{j}^{I V^{\prime \prime}}
$$

and

$$
\boldsymbol{f}_{i}^{I D V}=-\sum_{j}\left(\delta_{i j} \mathbf{1}-\boldsymbol{P}_{i j}\right) \cdot \boldsymbol{f}_{j}^{I V^{\prime}}
$$

Since

$$
\boldsymbol{f}_{i}^{I V^{\prime}}=-\boldsymbol{f}_{i}^{R}-\boldsymbol{f}_{i}^{I D V}=-\boldsymbol{f}_{i}^{B}-\boldsymbol{f}_{i}^{I R V}-\boldsymbol{f}_{i}^{I D V}
$$


the fundamental equation (23) may also be written as:

$$
\tilde{T}=\frac{1}{V} \sum_{i}\left(\boldsymbol{f}_{i}^{B}+\boldsymbol{f}_{i}^{I R V}+\boldsymbol{f}_{i}^{I D V}\right) \boldsymbol{r}_{i} .
$$

This expression can be split up in two different ways:

$$
\tilde{T}=\frac{-1}{V} \sum_{i}\left(f_{i}^{R}+\boldsymbol{f}_{i}^{I D V}\right) \boldsymbol{r}_{i}=\tilde{\boldsymbol{T}}^{R}+\tilde{\boldsymbol{T}}^{D}
$$

or equivalently

$$
\tilde{\boldsymbol{T}}=\frac{-1}{V} \sum_{i}\left(\boldsymbol{f}_{i}^{B}+\boldsymbol{f}_{i}^{I V}\right) \boldsymbol{r}_{i}=\tilde{\boldsymbol{T}}^{B}+\tilde{\boldsymbol{T}}^{I},
$$

in which $f_{i}^{I V}=f_{i}^{I D V}+f_{i}^{I R V}$, the total force due to the force fields between the particles and the constraining forces as well. In this way we also obtain a justification of the expression proposed in (21).

\section{Example}

In order to illustrate the concepts introduced in the preceding sections we now consider a specific example. To this end we take the well-known rigid dumbbell model. We consider a dilute system, so it is sufficient to treat one dumbbell, consisting of a rigid rod with two particles at is terminal points $\boldsymbol{r}_{1}$ and $\boldsymbol{r}_{2}$. The endto-end-vector will be denoted by $\boldsymbol{q}=\boldsymbol{r}_{2}-\boldsymbol{r}_{1}$ and the corresponding unit vector by $\boldsymbol{e}=\boldsymbol{q} /|\boldsymbol{q}|$.

In this case the constraint equation reads:

$$
C(\boldsymbol{q})=\boldsymbol{q} \cdot \boldsymbol{q}-\boldsymbol{q}^{2}=0, \quad(\boldsymbol{q}=|\boldsymbol{q}|=\text { constant }),
$$

so the gradient vector in configuration space becomes

$$
\frac{\partial C}{\partial\left\{\boldsymbol{r}_{1}, \boldsymbol{r}_{2}\right\}}=q\{-\boldsymbol{e}, \boldsymbol{e}\} \text {. }
$$

From this result, using eq. (19), the projection operator $\boldsymbol{P}_{i j}$ is calculated to be:

$$
\left[P_{i j}\right]=\left[\begin{array}{cc}
1-\frac{1}{2} e e & \frac{1}{2} e e \\
\frac{1}{2} e e & 1-\frac{1}{2} e e
\end{array}\right] .
$$

Although it is possible to continue the calculation with this projection operator, defined in the $\left\{\boldsymbol{r}_{1}, \boldsymbol{r}_{2}\right\}$ space, it is more instructive to use first a transformation to the $\left\{\boldsymbol{r}_{0}, \boldsymbol{q}\right\}$ space, in which $\boldsymbol{r}_{0}=\frac{1}{2}\left(\boldsymbol{r}_{1}+\boldsymbol{r}_{2}\right)$ is the centre of the dumbbell. After this transformation a new projection operator is obtained, given by the matrix:

$$
\left[\boldsymbol{P}_{i j}^{\prime}\right]=\left[\begin{array}{cc}
1 & 0 \\
\mathbf{0} & 1-e e
\end{array}\right] .
$$

Indeed, for an arbitrary variation $\left\{\delta \boldsymbol{r}_{0}, \delta \boldsymbol{q}\right\}$, multiplication with $\boldsymbol{P}_{i j}^{\prime}$ give $\left\{\delta \boldsymbol{r}_{0},(\mathbf{1}-\boldsymbol{e} \boldsymbol{e}) \cdot \delta \boldsymbol{q}\right\}$ which is the part of the variation allowable by the constraints, and multiplication with $\left(\delta_{i j} \mathbf{1}-\boldsymbol{P}_{i j}^{\prime}\right)$ gives $\{0, \boldsymbol{e} \boldsymbol{e} \cdot \delta \boldsymbol{q}\}$ which is a motion, prevented by the constraints.

The reversible contribution of the dumbbell to the stress tensor now becomes

$$
\tilde{\boldsymbol{T}}^{R}=-\left\langle\boldsymbol{P}_{i j} \cdot \boldsymbol{f}_{i}^{R} \boldsymbol{r}_{j}\right\rangle
$$

or, after transformation

$$
\tilde{\boldsymbol{T}}^{R}=-\left\langle\boldsymbol{f}_{0}^{R} \boldsymbol{r}_{0}\right\rangle-\left\langle(\mathbf{1}-\boldsymbol{e} \boldsymbol{e}) \cdot \boldsymbol{f}_{\boldsymbol{q}}^{R} \boldsymbol{q}\right\rangle
$$

in which

$$
f_{0}^{R}=-k T \frac{\partial}{\partial r_{0}} \ln \frac{\psi}{\psi_{0}}
$$

and

$$
f_{q}^{R}=-k T \frac{\partial}{\partial \boldsymbol{q}} \ln \left(\frac{\psi}{\psi_{0}}\right) .
$$

Since $\psi_{0}=$ constant and

$$
\frac{\partial}{\partial \boldsymbol{q}} \cdot(1-e e) \boldsymbol{q}=-(3 e \boldsymbol{e}-\mathbf{1})
$$

we obtain after partial integration:

$$
\tilde{\boldsymbol{T}}^{R}=-k T \mathbf{1}+k T\langle 3 \boldsymbol{e} \boldsymbol{e}-\mathbf{1}\rangle .
$$

The two terms in this expression are Brownian contributions: the first one is due to the translation and the second one to the rotational degree of freedom of the particle.

The dissipative part of the stress tensor becomes

$$
\tilde{\boldsymbol{T}}^{D}=\left\langle\left(\delta_{i j} \mathbf{1}-\boldsymbol{P}_{i j}\right) \cdot \boldsymbol{f}_{i}^{H} \boldsymbol{r}_{j}\right\rangle
$$

where in this case the external force is the hydrodynamic force $f_{i}^{H}=-\zeta\left(\boldsymbol{u}_{i}-\boldsymbol{v}_{i}\right)$, or after transformation

$$
\tilde{\boldsymbol{T}}^{D}=\left\langle\boldsymbol{e} \boldsymbol{e} \cdot \boldsymbol{f}_{\boldsymbol{q}}^{H} \boldsymbol{q}\right\rangle
$$

in which

$$
\boldsymbol{f}_{q}^{H}=-\frac{1}{2} \zeta q(\dot{\boldsymbol{e}}-\boldsymbol{L} \cdot \boldsymbol{e}) .
$$

So we have

$$
\tilde{T}^{D}=\frac{1}{2} \zeta q^{2}\langle\boldsymbol{e} \boldsymbol{e e e}\rangle: D
$$

with $\boldsymbol{D}=\frac{1}{2}\left(\boldsymbol{L}+\boldsymbol{L}^{\mathbb{T}}\right)$ the rate of strain tensor. From (66) and (70) we finally obtain the well known result:

$$
\tilde{\boldsymbol{T}}=-k T \mathbf{1}+k T\langle 3 \boldsymbol{e} \boldsymbol{e}-\mathbf{1}\rangle+\frac{1}{2} \zeta \boldsymbol{q}\langle\boldsymbol{e} \boldsymbol{e} \boldsymbol{e} \boldsymbol{e}\rangle: \boldsymbol{D} .
$$

This example shows in which way eqs. (56) and (57) for the stress tensor can be used in a systematic way and also the central role of the constraint operator $\boldsymbol{P}_{i j}$ in this treatment. 


\section{References}

1. Jongschaap RJJ, Doeksen D (1983) Rheol Acta 22:4

2. Bird RB, Curtiss CF (1985) J Polym Sci 73:187

3. Curtiss CF, Bird RB (1981) J Chem Phys 74, 2016, 2026

4. Curtiss CF, Bird RB (1983) Physica 118 A, 191

5. Curtiss CF, Bird RB, Hassager O (1976) Adv Chem Phys 35,31

6. Batchelor GK (1970) J Fluid Mech 41, 545

7. Brenner H (1970) Ann Rev Fluid Mech 2, 137

8. Irving JH, Kirkwood JG (1950) J Chem Phys 18, 817

9. Kirkwood JG (1949) Rec Trac Chim 68, 649

10. Fixman M, Kovac J (1978) J Chem Phys 61, 4939 (1974); 61,$4950 ; 63,935$
11. Doi, M (1983) J Chem Phys 79, 5080

12. De Groot SR, Mazur P (1969) "Non-Equilibrium Thermodynamics" Amsterdam-London p 126

13. Rouse PE Jr (1953) J Chem Phys 21:1272

(Received November 28, 1986)

Author's address:

Drs. R. J. J. Jongschaap

Dept. of Applied Physics

Twente University of Technology

Reology Group

P.O. Box 217

NL-7500 AE Enschede 